\title{
TRANSIENT COHERENT RAMAN SCATTERING IN THE TIME AND FREQUENCY DOMAIN
}

\author{
W. ZINTH \\ Physik Department der Tečhnischen Universität München, \\ Munchen, Fed. Rep. Germany
}

Received 2 June 1980

\begin{abstract}
A new type of Raman spectroscopy is presented: After transient excitation of molecular modes coherently scattered Raman spectra are investigated irr a delayed probing experiment. The spectral position of the Raman mode is observed after long delay times. The dephasing time is obtained from the time dependence of the scattered amplitudes. Frequency disturbing non-resonant susceptibilities are eliminated. We report on first experimental results of transient coherent Raman spectroscopy of liquid $\mathrm{CH}_{3} \mathrm{CCl}_{3}$.
\end{abstract}

\section{Introduction}

During the past years, coherent Raman spectroscopy has proven to be a valuable tool for the investigation of molecular vibrations in various surroundings. The analysis is difficult for stationary coherent Raman spectra since a non-resonant coherent background interferes with the vibrational signal [1]. The background is produced by the nonlinear reaction of the electrons following immediately an applied electric field (within $10^{-14} \mathrm{~s}$ ). The molecular vibrations producing the resonant signal, on the other hand, have a slower response time of $\sim 10^{-12} \mathrm{~s}$. We have demonstrated in a time resolved coherent experiment that the different time dependence may be used to separate resonant and non-resonant contributions to coherent Raman scattering [2]. Very recently, this idea has been applied. by Kamga and Sceats [3] who rejected the background in a transient coherent anti-Stokes Raman experiment.

We shall present here a different type of transient coherent Raman spectroscopy allowing to overcome the non-resonant background and to obtain new information on the molecular modes [4]. Two ultrashort light pulses at frequencies $\omega_{L}$ and $\omega_{S}$ excite molecular vibrations within a frequency band around $\omega_{L}-\omega_{S}$. After a certain delay time, where fast excitations have vanished, a third light pulse is applied to probe the remaining material excitation by coherent Raman scattering. The anti-Stokes or Stokes light is spectrally analyzed with high frequency resolution.

\section{Theory}

Coherent Raman scattering is treated in the transient regime where the molecular response time has the same magnitude as the pulse durations. We concentrate on anti-Stokes probing of a molecular vibration - the generalisation to the Stokes case is straightforward $[5,6]$.

The wave equation containing the nonlinear polarisation $P^{N L}$ describes the propagation of the light $E$. We split off $\boldsymbol{P}^{\mathrm{NL}}$ into a slow part due to the vibrations and a fast non-resonant part due to the nonlinear electronic motion $[2,6]$ :

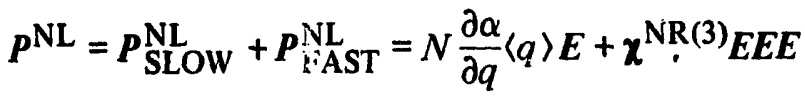

The fast component of the nonlinear polarisation is represented by the real, third order susceptibility $x^{\mathrm{NR}(3)}$. The coherent amplitude $\langle q\rangle$ - the expectation value of the vibrational mode operator - describes the reaction of the molecules. $\langle q\rangle$ obeys the equation of motion of a harmonic oscillator with resonance frequency $\omega_{0}$, damping constant $2 / T_{2}$, and a driving force proportional to $E^{2}[5,6,7] . N$ is the number density of the molecules with Raman jolarisability $\partial \alpha / \partial q$. In the present discussion we use plane waves with amplitudes $E_{j}$ and $Q$ for the electric fields and the coherent excitation, respectively. Using eq. (1) in the material and wave equations we obtain $[5,6,8]$ : 


$$
\begin{aligned}
& \frac{\partial Q}{\partial t^{*}}+Q\left(\frac{1}{T_{2}}+\mathrm{i}\left(\frac{\omega_{0}^{2} \cdots \omega_{\mathrm{v}}^{2}}{2 \omega_{\mathrm{v}}}\right)\right) \propto E_{\mathrm{L}} E_{\mathrm{S}}^{*} \\
& \frac{\partial E_{\mathrm{A} 2}}{\partial x^{\prime}} \propto\left\{\left(N \frac{\partial \alpha}{\partial q} E_{\mathrm{L} 2} Q+6 \chi_{\mathrm{LS}}^{\mathrm{NR}(3)} E_{\mathrm{L} 2} E_{\mathrm{L}} E_{\mathrm{S}}^{*}\right)\right. \\
& \left.\quad \times \exp \left(-\mathrm{i} \Delta k_{\mathrm{A} 2} x\right)+6 x_{\mathrm{LA} 2}^{\mathrm{NR}(3)}\left(\left|E_{\mathrm{L} 2}\right|^{2}+\left|E_{\mathrm{L}}\right|^{2}\right) E_{\mathrm{A} 2}\right\}
\end{aligned}
$$

A retarded time frame $x^{\prime}$ and $t^{\prime}$ was used. $T_{2}$ denotes the dephasing time of the molecular vibration.

$V_{i j}^{N R(3)}$ describes formally the action of the light at frequency $\omega_{\mathrm{i}}$ on the light at $\omega_{j}$ by way of the nonresonant susceptibility [8]. $\Delta k_{\mathrm{A} 2}=k_{\mathrm{A} 2}-k_{\mathrm{L} 2}$

$k_{\mathrm{L}}+k_{\mathrm{S}}$ represents the mismatch in wave-vector geometry. $\Delta k_{\mathrm{A} 2}=0$ is called phase matching.

Eq. (2) shows the excitation of the coherent amplitude by the laser and Stokes fields $E_{\mathrm{L}}$ and $E_{\mathrm{S}}$. The frequency spread of the incoming light pulses due to their short duration stimulates molecules within a bandwidth around the frequency difference $\omega_{v}=$ $\omega_{L} \quad \omega_{S}$. During the excitation process the molecules are driven by $\omega_{v}$. When the pumping fields have passed the medium, the molecules return to their resonance frequency $\omega_{0}^{\prime}$ and the coherent amplitude $Q$ decays exponentially with the dephasing time $T_{2}$. Production of coherent light near the anti-Stokes frequency $\omega_{\mathrm{A} 2}$ is determined by eq. (3). Under phasematching conditions one finds two important contributions to $E_{\mathrm{A} 2}$ :

(i) Scattering of the delayed probing light pulse $E_{\mathrm{L} 2}$ by the coherent amplitude $Q$ produces resonant anti-Stokes light at the frequency $\omega_{\mathrm{A} 2}=\omega_{\mathrm{L} 2}+\omega_{\mathrm{q}}$ where $\omega_{\mathbf{q}}$ is the momentary vibrational frequency of the molecules. The scattered energy is proportional to $\left|Q E_{\mathrm{L}_{2}}\right|^{2}$ and do ays exponentially at later delay times.

(ii) The non-resonant susceptibility produces antiStokes light when exciting and probing pulses have a temporal overlap in the sample. The respective antiStokes frequency is equal to $\omega_{\mathrm{A} 2}=\omega_{\mathrm{L} 2}+\omega_{\mathrm{L}} \cdots \omega_{\mathrm{S}}$. For sufficient delav +imes the non-resonant signal disappears.

The spectral pric serties of the coherently produced light may be strongly affected by phase modulation. For weak probing pulses $\left|E_{\mathrm{L} 2}\right| \ll\left|E_{\mathrm{L}}\right|,\left|E_{\mathrm{S}}\right|$ the main phase modulation results during the excitation process. it may be omitted at later delay times.

We discuss two types of transient coherent Raman spectroscopy. In both cases the excitation is made by two simultaneously applied ultrashort light pulses at frequencies $\omega_{L}$ and $\omega_{S}$. The probing process is performed at a delay time $t_{\mathrm{D}}$ after the peak of the exciting laser pulse. A first type of transient colerent Raman spectroscopy was recently reported in ref. [3], where the frequency difference $\omega_{L}-\omega_{S}$ of the excitation is tuned. The coherent anti-Stokes light generated in the delayed probing process is detected as a function of frequency $\omega_{L}-\omega_{S}$ with the non-reonant background being rejected. Some difficulties may arise with this technique: In the transient excitation process closely lying modes are simultaneously excited and all these modes are probed. As a consequence, the frequency resolution is limited by the excitation band. width and the spectrum may be modified by interfering modes [5,9].

We propose here a different type of transient coherent Raman spectroscopy: The excitation is performed at the frequency difference $\omega_{L}-\omega_{S}$ with very short light pulses (broadband excitation). In the probing process we use a smaller bandwidth (longer pulse) and observe the coherently scattered light spectrally resolved as a function of delay time. At small delay times we are able to see the influence of the driving process combined with the effects from the nonresonant susceptibility. Later on, the non-resonant background disappears and resonant molecular properties determine the signal. Interference may occur only within the probing bandwidth and may be controlled by measuring at different delay times. It should be noted that for suitably shaped probing pulses (gaussian shape) and long delay times, the bandwidth of the coherently scattered light is equal to the width of the probing pulse and may be smaller than the spontaneous Raman line.

\section{Experimental}

We investigated the $\mathrm{CH}_{3}$-stretching mode of liquid $\mathrm{CH}_{3} \mathrm{CCl}_{3}$ at $2939 \mathrm{~cm}^{-1}$. This transition is homogeneously broadened with a dephasing time $T_{2}=2.5 \mathrm{ps}$ [4,5]. This molecule appears to be well suited to test our theoretical predictions. The experimental set-up 


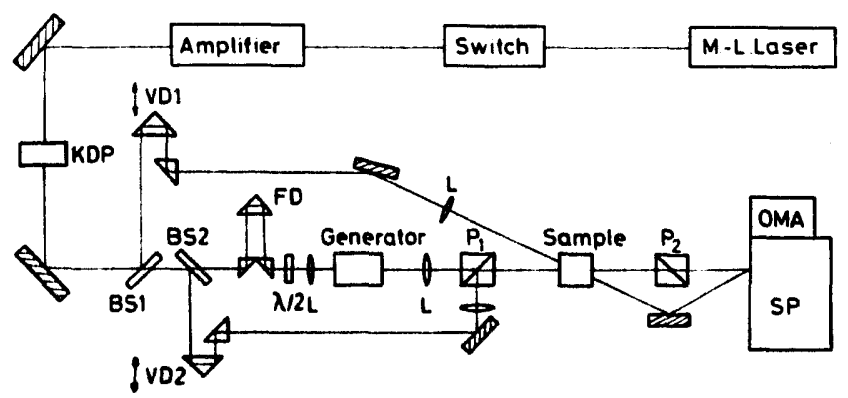

Fig. 1. Schematic of the experimental system to study transient colherent Stokes and anti-Stokes spectra. Second harmonic generator, KDP; beamsplitters, $\mathrm{BS}_{1}, \mathrm{BS}_{2}$; variable and fixed delay linus, $V D_{1}, V D_{2}$, and $F D ; \lambda / 2$ plate, $\lambda / 2$; lenses, $L$; polarizers, $P_{1}, P_{2} ; 2 \mathrm{~m}$ grating spectrograph, SP; optical multichannel analyzer, OMA.

is shown schematically in fig. 1 . A single chirp-free picosecond light pulse from a $\mathrm{Nd}$ : glass laser system is frequency-doubled in a KDP crystal to $\nu / c=$ $18980 \mathrm{~cm}^{-1}$ [10]. The pulse of duration 6.5 ps produces stimulated Stokes light in the generator cell filled with liquid $\mathrm{CH}_{3} \mathrm{CCl}_{3}[8,11]$. The Stokes conversion efficiency is approximately $5 \%$. The Stokes converpulse and generated Stokes pulses are imaged into the sample cell (length $1 \mathrm{~cm}$ containing $\mathrm{CH}_{3} \mathrm{CCl}_{3}$ ) and produce here the coherent material excitation. Weak probing pulses $E_{\mathrm{L} 2}$ and $E_{\mathrm{L} 2}$ are generated at the beam splitters $B_{1}$ and $B S_{2}$ properly delayed by the variable delays $V D_{1}$ and $V D_{2}$. The probing pulse for anti-Stokes scattering crosses the exciting beams in the sample cell under the phase matching angle of $7^{\circ}$. Interaction of the coherent excitation with the probing pulse produces the coherent anti-Stokes light. This light is imaged onto the slit of a $2 \mathbf{~ m}$ grating spectrograph, SP, and detected by an optical multichannel analyzer. The light beam for Stokes probing is coupled in at the polarizer $P_{1}$. It travels collinearly with the exciting beams through the sample. Here, the incoming beams produce the colierent Stokes light passing the polarizer $\mathbf{P}_{2}$. The coherent Stokes light is also detected by the spectrograph and the optical multichannel analyser. The spectrograph contains two gratings allowing simultancous measurements of Stokes and antiStokes spectra; the spectral resolution of the system is $0.5 \mathrm{~cm}^{-1}$.

\section{Results and discussion}

Fig. 2 shows coherent anti-Stokes spectra taken in $\mathrm{CH}_{3} \mathrm{CCl}_{3}$ at different delay times. Time zero in our experiments is determined by the maximum of the pumping laser pulse. In transient stimulated Raman scattering of $\mathrm{CH}_{3} \mathrm{CCl}_{3}$ the maximum of the coherent excitation occurs delayed by 5 ps. In fig. 2a we present a coherent anti-Stokes spectrum taken at $t_{\mathrm{D}}=$ $1.5 \mathrm{ps}$. It carries information on the rising part of the material excitation. We find a relatively broad spec$\operatorname{trum}\left(\Delta v / c \approx 5 \mathrm{~cm}^{-1}\right)$ with the center frequency at $21922 \mathrm{~cm}^{-1}$ corresponding to a Raman shift of 2942 $\mathrm{cm}^{-1}$. The second spectrum is taken at $t_{\mathrm{D}}=8.5 \mathrm{ps}$, shortly after the maximum of the coherent excitation. Here, the molecules start to vibrate their resonance

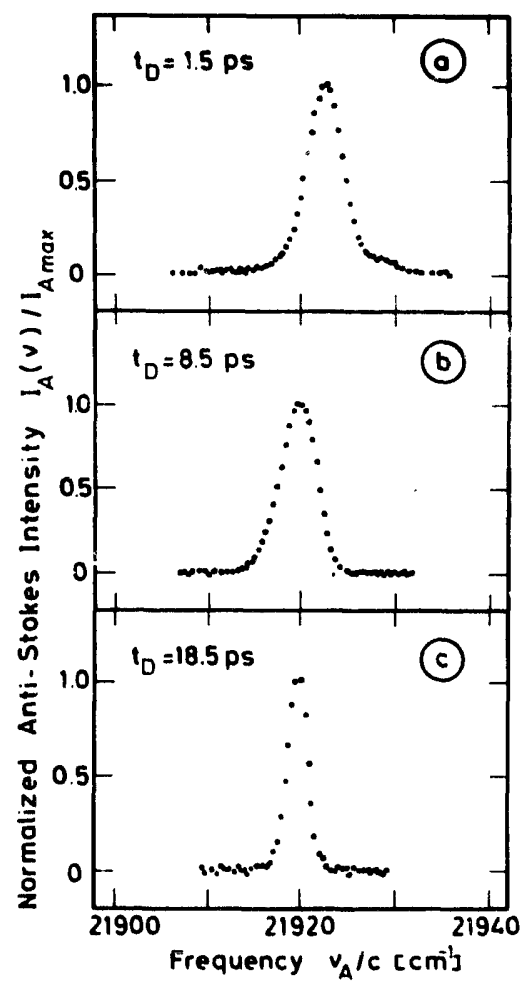

1.ig. 2. Transient coherent anti-Stokes spectra of the $\mathrm{CH}_{3}$ stretching mode of liquid $\mathrm{CH}_{3} \mathrm{CCl}_{3}$. The experimental spectra are measured at different delay times: fig. $2 a$ and fig. $2 b$ at ${ }^{t} \mathrm{D}=1.5 \mathrm{ps}$ and $8.5 \mathrm{ps}$ before and bchind the peak of the coherent excitation, respectively. Fig. $2 c$ is taken at a later delay time, $t_{\mathrm{D}}=\mathbf{1 8 . 5} \mathrm{ps}$, in the exponential decay of the material excitation. At that time, there is no overlap of exciting and probing light pulses. The spectrum is not influenced by the excitat. on process and reflects the pure molec ular properties. 
frequency. Again, the spectrum is broad, $\Delta v / c \approx 5$ $\mathrm{cm}^{-1}$. The peak of the spectrum has shifted by about $3 \mathrm{~cm}^{-1}$ to smaller frequencies. At even later delay times, $t_{D}=18.5 \mathrm{ps}$ (fig. $2 \mathrm{c}$ ), the spectrum is narrower and shows only small changes of the frequency position. The experimental results illustrate the importance of the different terms of eq. (3). Near the maximum of the coherent amplitude, the excitation process is . relevant (first term of the r.h.s. of eq. (3)). The coherent amplitude $Q$ is driven by the laser and Stokes field with the momentary frequency $\omega_{L}-\omega_{S}$ that may slightly differ from the resonance frequency $\omega_{0}[8]$. As the coherent amplitude varies rapidly the coherent light is produced during a short period with a broad spectrum. In addition, phase modulation due to the puimping laser (last term of eq. (3)) may shift the spectral position to higher frequencies. On the other hand, the true molecular properties are found at later delay times. We have measured simultaneously the coherent Stokes and anti-Stokes spectra and obtained a frequency of the coherently oscillating molecules of $2938.2 \pm 0.7 \mathrm{~cm}^{-1}$. This result is in full agreement with the sportaneous Raman shift of $2939.0 \pm 0.5$ $\mathrm{cm}^{-1}$.

In fig. 3 we compare the spontaneous Raman spectrum measured with a conventional arrangement (solid line) and a transient coherent anti-Stokes spectrum taken at $t_{\mathrm{D}}=18.5 \mathrm{ps}$ (points). The position of the maxima is the same but striking differences of the shapes and half width may be noticed: The spontaneous Stokes spectrum has approximately lorentzian shape with a half width of $4.3 \mathrm{~cm}^{-1}$. On the other hand. the coherently measured spectrum has the gauss-

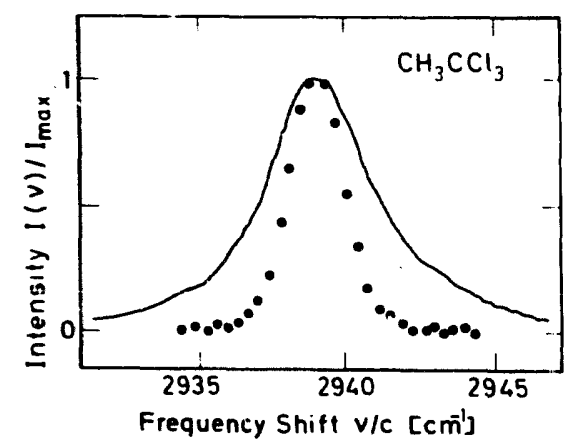

Fig. 3. Spontaneous Raman spectrum (solid line) and transient coh:rent spectrum (points) of the symmetric $\mathrm{CH}_{3}$-stretching mode in liquid $\mathrm{CH}_{3} \mathrm{CCl}_{3}$. The spectra show the same Raman shift $\left(2939 \mathrm{~cm}^{-1}\right)$ but different half widths of $4.3 \mathrm{~cm}^{-1}$ and $2.3 \mathrm{~cm}^{-1}$ for the spontaneous and the transient coherent spectrum, respectively. ian shape of the probing laser pulse and a narrower width of $2.3 \mathrm{~cm}^{-1}$. With our technique we obtain the spectral position directly. We measure the dephasing time $[5,6]$ and thus we have the fu'! information. Disturbing effects known from stationary coherent Raman scattering are not present with our technique.

We have presented a new type of coherent Raman spectroscopy. This technique shows a number of promising possibilities: The non-resonant background is negligible and - as a consequence - coherent spectra of diluted systems become possible. Spectral positions and molecular time constants can be measured simultaneously with high accuracy. In the present experiment, the frequency difference of the exciting pulses is held constant and valuable information is obtained from the (high resolution) spectra of the coherently scattered radiation. More general fre. quency tuning of the input pulses in conjunction with the high resolution probing should allow to unravel complicated vibrational spectra.

\section{Acknowledgement}

The author gratefully acknowledges valuable collaboration of Professors W. Kaiser and A. Laubereau.

\section{References}

[1] P.D. Maker and R.W. Terhune, Phys. Rev. 137 (1965) A801; J.J. Song, G.L. Eesley and M.D. Leveson, Appl. Phys. Lett. 29 (1976) 567.

[2] W. Zinth, A. Laubereau and W. Kaiser, Optics Comm. 26 (1978) 457.

[3] F.M. Kamga and M.G. Sceats, Optics Lett. 5 (1980) 126.

[4] W. Zinth, Thesis, Technische Universität München, July 1979.

[5] A. Laubereau and W. Kaiser, Rev. Mod. Phys. 50 (1978) 607.

[6] A. Penzkofer, A. Laubereau and W. Kaiser, Progress in Quantum Electron. 6 (1979) 55.

[7] J.A. Giordmaine and W. Kaiser, Phys. Rev. 144 (1966) 676.

[8] W. Zinth and W. Kaiser, Optics Comm. 32 (1980) 507.

[9] A. Laubereau, G. Wochner and W. Kaiser, Phys. Rev, A13 (1976) 2212.

[10] W. Zinth, A. Laubercau and W. Kaiser, Optics Comm. 22 (1977) 161.

[11] S.A. Akhmanov, Mater. Res. Bull. 4 (1969) 455; R.L. Carman, F. Shimizu, C.S. Wang and N. Bloembergen, Phys. Rev. A2 (1970) 60. 